\title{
Boundary diffraction of an inhomogeneous wave
}

\author{
Pierre-A. Belanger \\ Laboratoire de Recherches en Optique et Laser, Département de Physique, Université Laval, Québec G1K 7P4, \\ Canada \\ Marc Couture \\ Collège d'Enseignement Genéral et Professionnel de Rimouski 60, de l'Evêcher Ouest, Rimouski, Canada
}

Received August 24, 1982; revised manuscript received November 20, 1982

\begin{abstract}
An exact decomposition of the diffracted field into a direct wave and a boundary diffraction wave is obtained for an incident inhomogeneous wave, namely, the complex-source-point spherical wave. Our result, in the paraxial approximation, is consistent with already published results on the diffraction of a Gaussian beam.
\end{abstract}

\section{INTRODUCTION}

The idea of the boundary-diffraction wave is primarily due to Sir Thomas Young, who suggested in $1801^{1}$ that diffraction caused by the presence of an obstacle in the path of light could be considered interference between the direct light and light reflected at each point of the boundary of the diffracting body. Young's ideas about diffraction were expressed only in a qualitative manner and were soon forgotten, as the morequantitative Huygens-Fresnel model of secondary waves began to dominate the field.

Nevertheless, the strong appeal of Young's model led some authors to investigate further in that direction. Maggi ${ }^{2}$ and Rubinowic $z^{3}$ independently demonstrated that, in the case of diffraction by an aperture in an opaque screen, the Kirchhoff integral (a mathematical formulation of the HuygensFresnel physical model) leads, for plane or spherical waves impinging upon the aperture, to a decomposition of the type suggested by Young, called the boundary-diffraction-wave (BDW) theory.

Some years ago, Miyamoto and Wolf ${ }^{4,5}$ obtained a generalization of that theory. They noted that the integrand appearing in the Kirchhoff diffraction formula can be written as the curl of a potential vector $W(P, Q)$, where $P$ is the observation point and $Q$ is an (unspecified) point in the aperture. Thereafter they showed that the diffracted field at point $P$, $U(P)$, may be expressed as the sum of two terms. The first, $U^{B}(P)$, is the contour integral of $\mathbf{W}$ along the boundary of the aperture. The second, $U^{S}(P)$, is shown to reduce to the sum of line integrals on infinitesimal paths surrounding the discontinuities of $\mathbf{W}$ in the aperture. They were able to obtain a general expression for the vector potential $W$ and to show that $U^{S}(P)$ is indeed equal to the direct (unperturbed) incident field for the cases already discussed by Maggi and Rubinowicz as well as in the case of a general incident wave in the geometrical-optics approximation.

It is important to realize, as these authors suggest elsewhere, ${ }^{6}$ that this alternative approach is not only of academic interest. Replacing double integrals with contour integrals can be quite time saving, especially if one considers today's complicated cascaded optical systems or related ones, such as optical resonators.

The purpose of the present paper is to present an exact decomposition of the diffracted field, for an inhomogeneous wave this time: the complex-source-point spherical wave (CSPSW). The CSPSW is a spherical wave whose origin is a point with complex coordinates rather than real ones. Its expression is easily shown to be an exact solution of the Helmholtz equation. This solution was first mentioned by Deschamps, ${ }^{7}$ and its properties were extensively analyzed later by Felsen. ${ }^{8}$ It was shown recently ${ }^{9}$ that the CSPSW can be viewed as the result of the summation of all the corrections to the paraxial Gaussian beam. This implies that the present work is directly related to the problem of diffraction of a laser beam by an aperture.

In Section 2 we derive an expression for the vector potential W associated with the CSPSW. Then, in Section 3, we find the locations of the singularities of $\mathbf{W}$ in the plane of the aperture and discuss the question of the lit and shadowed regions in the diffraction plane. In Section 4, the contribution from the integrals around these singularities is shown to give exactly the unperturbed incident field. In Section 5, we compare our exact result with those of Otis ${ }^{10}$ and Takenaka et al., ${ }^{11,12}$ who studied the diffraction of a Gaussian beam in the geometrical-optics approximation.

\section{VECTOR POTENTIAL $W(P, Q)$}

As was explained earlier, according to the BDW theory the diffracted field $U(P)$ at an observation point $P$ (Fig. 1) can be split into two parts:

$$
U(P)=U^{B}(P)+U^{S}(P) .
$$

The first term on the right-hand side of Eq. (2.1), $U^{B}(P)$, is a contribution from the edge of the diffracting aperture $\Gamma$. Its complete expression [see Ref. 5, Eq. (2.6)] is

$$
U^{B}(P)=\oint_{\Gamma} \mathrm{W}(P, Q) \cdot \mathrm{d} \mathbf{l}
$$




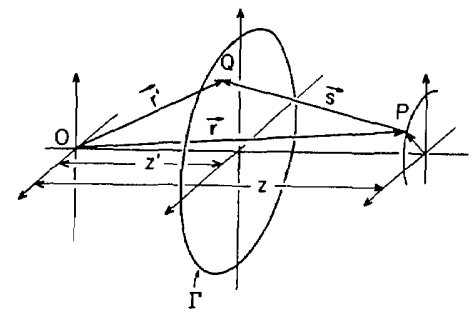

Fig. 1. Illustrating notation relating to the diffracted field at point $P$ by an aperture $\Gamma$ located in the plane $z^{\prime}$ from the origin $O$.

where the integration is made counterclockwise (viewed from $P$ ) and where the vector potential W [see Ref. 4, Eq. (4.15)] is

$$
\begin{aligned}
\mathbf{W}(P, Q) & =\mathbf{W}\left(\mathbf{r}, \mathbf{r}^{\prime}\right) \\
& =\mathbf{W}_{\infty}+\frac{e^{i k s}}{4 \pi s} \hat{s} \int_{\infty}^{0} e^{i k \mu} \nabla U^{i}\left(\mathbf{r}^{\prime}+\mu \hat{s}\right) \mathrm{d} \mu,
\end{aligned}
$$

$U^{i}$ being the incident field and $\hat{s}$ a unit vector in the direction of $\mathbf{s}=\mathbf{r}^{\prime}-\mathbf{r}$. The term $\mathbf{W}_{\infty}$ is discussed later.

The other term $U^{S}(P)$ arises from the singularities of $\mathbf{W}(P$, $Q)$ in the aperture and is discussed in Section 3. Here we take the incident field $U^{i}$ to be the CSPSW of Ref. 7,

$$
U^{i}(\mathbf{r})=\frac{\exp \left[i k R_{c}(\mathbf{r})\right]}{R_{c}(\mathbf{r})},
$$

where $R_{c}$ is the complex length of the vector $\mathbf{R}_{c}$, that is, ${ }^{13}$

$$
\begin{aligned}
R_{c} & =\left(\mathbf{R}_{c} \cdot \mathbf{R}_{c}\right)^{1 / 2}, \\
\mathbf{R}_{c}(\mathbf{r}) & =\mathbf{r}-i z_{0} \hat{z} .
\end{aligned}
$$

This expression is a solution of the Helmholtz equation obeying the Sommerfeld radiation condition. According to Miyamoto and Wolf, 4 for such a wave the term $\mathbf{W}_{\infty}$ in the expression for $\mathbf{W}$ is equal to zero. The remaining part of that expression is evaluated by first calculating $\nabla U^{i}$ :

$$
\nabla U^{i}\left(\mathbf{r}^{\prime}+\mu \hat{s}\right)=\mathbf{R}_{c}\left(i k-\frac{1}{R_{c}}\right) \frac{\exp \left(i k R_{c}\right)}{R_{c}^{2}},
$$

and Wolf [see Ref. 4, Eq. (5.10)] with the complex vector $\mathbf{R}_{c}\left(\mathbf{r}^{\prime}\right)$ replacing the real vector $\mathbf{r}^{\prime}$. However, as the complex nature of the radius $R_{\mathrm{c}}$ changes the characteristics of the spherical wave profoundly, making it nonuniform, the singularities of $\mathbf{W}$ will be considerably different from those of the regular spherical wave. This point is examined in Section 3.

\section{SINGULARITIES OF W IN THE APERTURE}

The second term $U^{S}(P)$ contributing to the total diffracted field is made of the sum of line integrals on paths encircling the points of discontinuity of W [see Ref. 5, Eq. (2.7)] in the aperture

$$
U^{S}(P)=U^{S}(\mathbf{r})=\sum_{j} \oint_{\Gamma_{j}} \mathbf{W}\left(\mathbf{r}, \mathbf{r}^{\prime}\right) \cdot \mathrm{d} \mathbf{l},
$$

where the $\Gamma_{j}$ 's are infinitesimal circular paths around the points of discontinuity of $\mathbf{W}$, the integration being made clockwise when viewed from $P$.

The locations of these discontinuities are obtained by making the denominator of the expression of $\mathbf{W}\left(\mathbf{r}, \mathbf{r}^{\prime}\right)$ equal zero, that is,

$$
R_{c}\left(\mathbf{r}^{\prime}\right)=0
$$

or

$$
R_{c}\left(\mathbf{r}^{\prime}\right)+\hat{s} \cdot \mathbf{R}_{\mathrm{c}}\left(\mathbf{r}^{\prime}\right)=0 .
$$

The singularity resulting from Eq. (3.2) corresponds to the singularity in the incident field itself and is excluded from the present analysis by putting the plane of the aperture on the right-hand side of the plane $z=0$, where this singularity lies. ${ }^{14}$

The other singularity, described by Eq. (3.3), is completely different from that of the regular spherical wave. In the latter case, the location of the singularity is the point where a straight line going from the origin to the observation point, called the direct ray, intersects the plane of the aperture. This is not true in the present case. To find the new location of the singularitv one has to realize that Fo. (3.3). containing com- 
and (3.5) become, respectively,

$$
\begin{gathered}
\rho^{\prime 2} z+\rho^{2} z^{\prime}-\rho \rho^{\prime}\left(z+z^{\prime}\right) \cos \left(\phi-\phi^{\prime}\right)=0, \\
\left(\rho \rho^{\prime}\right)^{2} \sin ^{2}\left(\phi-\phi^{\prime}\right)+\left(\rho z^{\prime}\right)^{2}+\left(\rho^{\prime} z\right)^{2}-2 z z^{\prime} \rho \rho^{\prime} \cos \left(\phi-\phi^{\prime}\right) \\
=z_{0}^{2}\left[\rho^{\prime 2}+\rho^{2}-2 \rho \rho^{\prime} \cos \left(\phi-\phi^{\prime}\right)\right] .
\end{gathered}
$$

These two equations can be combined and, after tedious but straightforward calculation, shown to define two lines of singularity, analogous to the direct rays mentioned earlier, for each observation point $P$. The singularities are again located at the intersection between these lines and the plane of the aperture.

These two lines lie on the same hyperboloid of revolution whose annular focus is a circle of radius $z_{0}$ located in the plane $z=0$. This hyperboloid is obtained by eliminating the trigonometric functions in Eq. (3.7) with the help of Eq. (3.6). The resulting equation is the equation of the hyperboloid but with its parameters evaluated in the plane $z^{\prime}$. The same hyperboloid is more simply written with the aid of Eqs. (3.8) and (3.9):

$$
\frac{\rho^{2}}{a^{2}}-\frac{z^{2}}{z_{0}^{2}-a^{2}}=1
$$

The specification of the observation point determines the parameter $a$; in turn, the distance $\rho^{\prime}$ from the axis to the point where the lines of singularity intersect the plane of the aperture located at $z^{\prime}$ is evaluated with ${ }^{15}$

$$
\frac{\rho^{\prime 2}}{a^{2}}-\frac{z^{\prime 2}}{z_{0}^{2}-a^{2}}=1 \text {. }
$$

Furthermore, these lines do not follow constant-angle paths on the hyperboloid. For any position along $z$ axis, they are symmetrically located on each side of the constant $-\phi$ line going through $P$; their angle $\phi^{\prime}$ in the plane of the aperture is determined by Eq. (3.6), namely,

$$
\cos \left(\phi-\phi^{\prime}\right)=\frac{\rho^{\prime 2} z+\rho^{2} z^{\prime}}{\rho \rho^{\prime}\left(z+z^{\prime}\right)}
$$

which gives two values that are equal but in opposite sign for the relative angle $\alpha=\phi-\phi^{\prime}$. This angle may be shown always to be less than or equal to $\pi / 2$, as the observation point recedes to infinity.

Figure 2 illustrates the two lines of singularity $\left(P Q_{1}\right.$ and $P Q_{2}$ ) associated with an observation point as well as the two points of singularity of $\mathbf{W}$ in the aperture $\left(Q_{1}\right.$ and $\left.Q_{2}\right)$.

With these results it is now easy to identify the lit and shadowed regions in the diffraction area. The shadowed re-

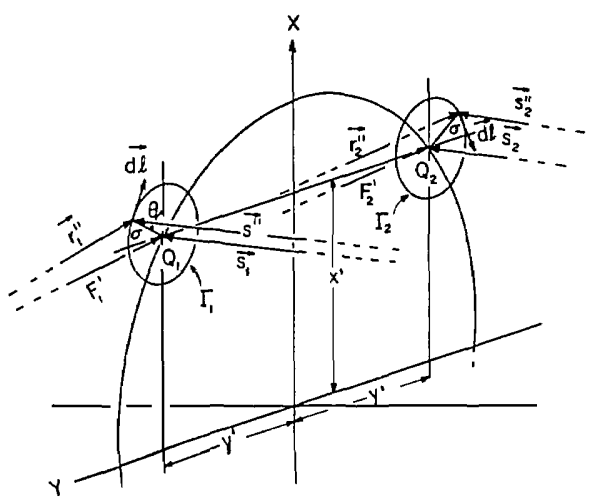

Fig. 3. Illustrating notation for the integration around infinitesimal circular contours $\Gamma_{1}$ and $\Gamma_{2}$ around the singularity $Q_{1}$ and $Q_{2}$. gion is defined as the region in space where an observation point defines no singularity of $\mathbf{W}$ inside the aperture; otherwise it is called the lit region. For an aperture with circular symmetry, the boundary of the two regions is well defined and corresponds to a hyperboloid of the type mentioned earlier, which coincides with the edge of the aperture in the $z^{\prime}$ plane. Note that this boundary does not exactly coincide, as predicted by geometrical optics, ${ }^{4}$ with a normal to the surfaces of constant phase of the wave. However, it may be shown that, as soon as one considers points some wavelengths away from the annulus of singularity of the CSPSW (located at $z$ $=0, \rho=z_{0}$ ), the two nearly coincide.

\section{CONTRIBUTION FROM THE SINGULARITIES}

We now calculate the contribution $U^{S}(P)$ from the singularities to the total diffracted field in the lit region. We must use Eq. (3.1), which reads now

$$
U^{S}(\mathbf{r})=\oint_{\mathrm{r}_{1}} \mathrm{~W}\left(\mathbf{r}, \mathbf{r}^{\prime}\right) \cdot \mathrm{d} \mathbf{l}+\oint_{\mathrm{r}_{2}} \mathrm{~W}\left(\mathbf{r}, \mathbf{r}^{\prime}\right) \cdot \mathrm{d} \mathbf{l},
$$

where $\Gamma_{1}$ and $\Gamma_{2}$ are infinitesimal circular contours of radius $\sigma$ around points of singularity $Q_{1}$ and $Q_{2}$ (Fig. 3).

If one recalls expression (2.8) for $W$, one finds that one has to evaluate, to first order in $\sigma$, the expressions

$$
N_{1,2} \equiv \mathrm{d} \mathbf{l} \cdot\left[\mathbf{s}_{1,2}^{\prime \prime} \times \mathbf{R}_{c}\left(\mathbf{r}_{1,2}^{\prime \prime}\right)\right] \quad \text { (numerator) }
$$

and

$$
\begin{aligned}
& D_{1,2} \equiv \mathbf{s}_{1,2}^{\prime \prime} R_{c}\left(\mathbf{r}_{1,2}^{\prime \prime}\right)\left[\mathbf{s}_{1,2}^{\prime \prime} R_{c}\left(\mathbf{r}_{1,2}^{\prime \prime}\right)+\mathbf{s}_{1,2}^{\prime \prime} \cdot \mathbf{R}_{c}\left(\mathbf{r}_{1,2}^{\prime \prime}\right)\right] \\
& \text { (denominator). }
\end{aligned}
$$

Note that both expressions have been multiplied by $s_{1,2}^{\prime \prime}$. After some calculation, one finds to first order that

$$
\begin{aligned}
N_{1,2}=\sigma \mathrm{d} \theta\left\{\left[\left(z-i z_{0}\right) x^{\prime}\right.\right. & \left.-\left(z^{\prime}-i z_{0}\right) \rho\right] \cos \theta \\
& \left. \pm\left(z-i z_{0}\right) y^{\prime} \sin \theta\right\}+O\left(\sigma^{2}\right) \mathrm{d} \theta
\end{aligned}
$$

and

$$
\begin{aligned}
D_{1,2}=\sigma R_{c}(\mathbf{r})\left\{\left[\left(x^{\prime}-\rho\right) R_{c}(\mathbf{r})\right.\right. & +s \rho] \cos \theta \\
& \left. \pm y^{\prime} R_{c}(\mathbf{r}) \sin \theta\right\}+0\left(\sigma^{2}\right),
\end{aligned}
$$

where in both cases $+(-)$ is for $2(1)$ and where the following relation has been used:

$$
R_{\mathrm{c}}(\mathbf{r})=R_{\mathrm{c}}\left(\mathbf{r}^{\prime}\right)+s .
$$

Each of the two contributions to $U^{S}(\mathbf{r})$ can now be written as

$$
\begin{aligned}
\lim _{\sigma \rightarrow 0} \oint_{\mathbf{r}_{1,2}} W\left(\mathbf{r}, \mathbf{r}_{1}^{\prime \prime}\right) \cdot \mathrm{d} \mathbf{l} & =\frac{\exp \left[i k R_{c}(\mathbf{r})\right]}{4 \pi R_{c}(\mathbf{r})} \\
& \times \int_{0}^{2 \pi} \frac{A \cos \theta \pm B \sin \theta}{C \cos \theta \pm D \sin \theta} \mathrm{d} \theta,
\end{aligned}
$$

where

$$
\begin{aligned}
& A=\left(x^{\prime}-\rho\right)\left(z-i z_{0}\right)+\left(z-z^{\prime}\right) \rho, \\
& B=y^{\prime}\left(z-i z_{0}\right), \\
& C=\left(x^{\prime}-\rho\right) R_{c}(\mathbf{r})+s \rho, \\
& D=y^{\prime} R_{c}(\mathbf{r}),
\end{aligned}
$$

and $+(-)$ corresponds to $2(1)$ again. 
Both integrals are readily evaluated by means of the residue theorem (see Appendix $\mathrm{A}$ ) and using the relations $A=i D$ and $B=-i C$; each of them is shown to equal $2 \pi$. We thus finally obtain the expected result:

$$
U^{S}(\mathbf{r})=\frac{\exp \left[i k R_{c}(\mathbf{r})\right]}{R_{c}(\mathbf{x})} .
$$

We therefore conclude that, when the observation point is in the lit region, the contributions from the two points of singularity of $\mathbf{W}$ in the aperture to the diffracted field add up exactly to yield the unperturbed incident field.

\section{DISCUSSION}

We have thus extended the results of BDW to a more-general case (which encompasses the spherical wave as a special case, e.g., when $z_{0}$ equals zero), the inhomogeneous CSPSW. This wave is also asymptotically equivalent, in the paraxial domain, to the well-known Gaussian beam. ${ }^{9}$

Otis ${ }^{10}$ considered the application of BDW theory to such a beam and obtained a result equivalent to ours in the paraxial domain. In particular, he found that the shadow boundary is given by a surface that coincides with the edge of the aperture and remains always perpendicular to the constant-phase surfaces of the wave, following hyperbolas. However, Otis ${ }^{9}$ seems to have found only one singularity in the aperture, instead of two as we did.

In two others papers, Takenaka et al., ${ }^{11,12}$ examining the same problem, indeed found two singularities in the aperture. The locations of these singularities exactly match those that we obtained if we use the approximation $\rho \ll\left|z-i z_{0}\right|$, clearly of a paraxial nature, in Eqs. (3.8)-(3.10).

The result of Otis ${ }^{10}$ is easily explained if one notes that the complex singularity around which he makes a contour integration is equivalent to two integrations in real space.

For the case of a spherical wave (or a plane wave) and also in the geometrical-optics approximation, ${ }^{4,5}$ the singularities in the aperture of the vector potential $W$ are exactly those points that lie on the rays that pass through $P$. For the CSPSW the interpretation is not straightforward. Even in the paraxial approximation, in which the CSPSW becomes the regular Gaussian beam for which the geometrical rays are the mentioned hyperbolas, it cannot be said that the singularities in the aperture lie on the rays that pass through $P$ unless one defines the rays as our lines of singularity that rotate while propagating. This double singularity of the vector potential $\mathbf{W}$ renders the separation between the lit and the shadowed boundary less drastic. For instance, a noncircular-symmetric aperture is such that for a certain region in space only one singularity is seen at point $P$. For this region only half of the direct wave contributes to the total field. The shadow boundary is somewhat ambiguous in real space for the inhomogeneous waves, such as the CSPSW.

\section{CONCLUSION}

Using the generalization of the boundary-diffraction theory of Miyamoto and Wolf, 4,5 we have obtained an exact decomposition of the diffracted field in a direct wave and a boundary wave when the incident field is the complex-source-point spherical wave. Our result contains all the known exact de- composition in boundary-diffraction-wave theory and could be generalized to another incident field, such as a complexsource-point sinusoidal wave, $\sin k R_{c} / R_{c}$. This last field is also paraxially equivalent to the usual Gaussian beam and does not contain the discontinuity of the complex-sourcepoint spherical wave.

\section{APPENDIX A}

In this appendix we evaluate the integral of Eq. (4.7), namely,

$$
F=\int_{0}^{2 \pi} \frac{A \cos \theta \pm B \sin \theta}{C \cos \theta \pm D \sin \theta} \mathrm{d} \theta,
$$

where $A=i D$ and $B=-i C$.

When the usual substitution $z=e^{i \theta}$ is made, the integral becomes a contour integral along unit circle in the complex plane. It may be written as

$$
F=\frac{1}{i} \oint \frac{(\mp C+i) z^{2} \pm C+i D}{(C \mp i D) z^{2}+C \pm i D} \frac{\mathrm{d} z}{z} .
$$

The integrand possesses three simple poles located at $z_{1}=$ 0 and at the two values $z_{2,3}$ of

$$
z_{2,3}=\left(-\frac{C \pm i D}{C \mp i D}\right)^{1 / 2} \text {. }
$$

However, it can be shown that the following relation:

$$
|C+i D|>|C-i D|
$$

always holds for $z>z^{\prime}$ so that, for the upper signs, the poles $z_{2}$ and $z_{3}$ lie outside the unit circle. The only pole contributing to the integral is thus $z_{1}$, and the residue at that point is easily evaluated:

$$
\operatorname{Res}_{z=0}=1 .
$$

On the other hand, all three poles contribute to the integral when the lower signs are considered. The residues are found to be

$$
\begin{aligned}
\operatorname{Res}_{z=0} & =-1, \\
\operatorname{Res}_{z=z_{2}} & =\operatorname{Res}_{z=z_{3}}=1 .
\end{aligned}
$$

The integral being equal to $2 \pi i$ times the sum of the residues located inside the contour of integration, one sees that its value is $2 \pi$ for both choices of signs.

\section{ACKNOWLEDGMENT}

This work was made possible by the financial support of the Ministère de l'Education du Québec and of the National Research Council of Canada.

\section{REFERENCES}

1. T. Young, "Lectures on natural philosophy, diffraction," Phil. Trans. R. Soc. London 92, 12-49 (1802).

2. G. A. Maggi, Ann. Mat. IIa, 16, 21 (1888).

3. A. Rubinowicz, "Die Beugungswelle in der Kirchhoffschen Theorie des Beugungsercheinungen," Ann. Phys. 53, 257-278 (1917).

4. K. Miyamoto and E. Wolf, "Generalization of the Maggi-Rubinowicz theory of the boundary diffraction wave-Part I," J. Opt. Soc. Am. 52, 615-625 (1962). 
5. K. Miyamoto and E. Wolf, "Generalization of the Maggi-Rubinowicz theory of the boundary diffraction wave-Part II," J. Opt. Soc. Am. 52, 626-637 (1962).

6. E. Wolf, "Some recent research on diffraction of light," in Modern Optics (Polytechnic Institute of Brooklyn, New York, 1967), pp. 433-453.

7. G. A. Deschamps, "Gaussian beam as a bundle of complex rays," Electron. Lett. 7, 684-685 (1971).

8. L. B. Felsen, "Complex-source-point solutions of the field equations and their relation to the propagation and scattering of Gaussian beams," in Symposia Mathematica (Academic, New York, 1976), Vol. XVIII.

9. M. Couture and P. A. Belanger, "From Gaussian beam to complex-source-point-spherical wave," Phys. Rev. A 24, 355-359 (1981).

10. G. Otis, "Application of the boundary-diffraction wave theory to Gaussian beams," J. Opt. Soc. Am. 64, 1545-1550 (1974).

11. T. Takenaka, M. Kakeya, and O. Fukumitsu, "Asymptotic representation of the boundary-diffraction wave for a Gaussian beam incident on a circular aperture," J. Opt. Soc. Am. 70, 1323-1328 (1980).

12. T. Takenaka and O. Fukumitsu, "Asymptotic representation of the boundary-diffraction wave for a three-dimensional Gaussian beam incident upon a Kirchhoff half-screen," J. Opt. Soc. Am. 72, 331-336 (1982).

13. $z_{0}$ is the Rayleigh range $\pi W_{0}^{2} / \lambda$ of the corresponding paraxial Gaussian beam of beam waist $W_{0}$.

14. The case in which the plane of the aperture is on the left-hand side of the plane $z=0$ could also be analyzed in a way similar to that for a regular converging spherical wave.

15. Note that, in order that Eqs. (3.4) and (3.5) be satisfied, $a$ must always be smaller than $z_{0}$. 